\title{
Anti-osteoporotic effects of tetramethylpyrazine via promoting osteogenic differentiation and inhibiting osteoclast formation
}

\author{
LONG WANG* ${ }^{*}$,WEI-GUANG LU*, JUN SHI*, HONG-YANG ZHANG, XIAO-LONG XU, BO GAO, \\ QIANG HUANG, XIAO-JIE LI, YA-QIAN HU, QIANG JIE, ZHUO-JING LUO and LIU YANG
}

Institute of Orthopedic Surgery, Xijing Hospital, The Fourth Military Medical University, Xi'an, Shaanxi 710032, P.R. China

Received January 16, 2017; Accepted August 3, 2017

DOI: $10.3892 / \mathrm{mmr} .2017 .7610$

\begin{abstract}
Long-term glucocorticoid therapy results in various side effects, including a high incidence of glucocorticoid-induced osteoporosis (GIOP), which is the most common form of secondary osteoporosis. Excess glucocorticoids reduce the viability of bone marrow-derived mesenchymal stem cells (BMSCs) and prolong osteoclast survival. These two types of cell are essential in the balance between bone formation and resorption. Tetramethylpyrazine (TMP), the pharmacologically active component extracted from Chuanxiong, has been reported to protect BMSCs from glucocorticoid-induced apoptosis. In the present study, the protective effects of TMP on BMSC differentiation and osteoclasts maturation in GIOP were investigated in vivo and in vitro. The immunostaining of osterix (OSX) and tartrate-resistant acid phosphatase (TRAP) staining indicated that TMP promoted osteogenesis and inhibited osteoclastogenesis in a rat model of GIOP. Treatment with $10^{-6} \mathrm{M}$ dexamethasone (Dex) significantly inhibited BMSC differentiation and increased TRAP-positive cells in vitro. However, different concentrations of TMP (50, 100 and $200 \mu \mathrm{M}$ ) ameliorated the negative effects of Dex by promoting the activity of alkaline phosphatase (ALP) and the calcium mineralization of BMSCs following osteogenic induction, which increased the expression levels of osteogenic genes, including ALP, collagen type I $\alpha 1$, osteocalcin and OSX, and decreased osteoclastogenesis-related genes, including TRAP, nuclear factor of T-cells cytoplasmic 1 and cathepsin K. In addition, it was found that the inhibition of receptor activator of nuclear factor- $\kappa \mathrm{B}$ ligand and intereleukin- 6 in BMSCs may
\end{abstract}

Correspondence to: Professor Liu Yang or Professor Zhuo-Jing Luo, Institute of Orthopedic Surgery, Xijing Hospital, The Fourth Military Medical University, 15 Changle West Road, Xi'an, Shaanxi 710032, P.R. China

E-mail: yangliu@fmmu.edu.cn

E-mail: zjluofmmu@hotmail.com

*Contributed equally

Key words: tetramethylpyrazine, glucocorticoids, osteoporosis, mesenchymal stem cells, osteoclasts be a possible mechanism for the protective effects of TMP against glucocorticoid-induced osteoclastogenesis. These results are the first, to the best of our knowledge, to demonstrate that TMP promotes BMSC differentiation and inhibits osteoclastogenesis to ameliorate bone mass change in GIOP.

\section{Introduction}

Glucocorticoid-induced osteoporosis (GIOP) is the most common form of secondary osteoporosis due to the wide use of glucocorticoid therapy in clinical practice $(1,2)$. Long-time glucocorticoid treatment leads to early and rapid bone loss, and increased fracture risk. GIOP predominantly affects the skeletal regions containing abundant cancellous bone, including the lumbar spine and proximal femur (3). The bone loss caused by glucocorticoids results from their direct effects on osteoblasts, osteoclasts and osteocytes. Previous studies have shown that excess glucocorticoids inhibit the proliferation and increase the apoptosis of osteoblasts and osteocytes (4), and prolong osteoclast survival (5).

It is well known that bone tissue in adult mammalian animals undergoes continuous remodeling through a strictly controlled balance between bone formation and resorption. This dynamic balance of physiological bone mass is maintained constantly by osteoblasts and osteoclasts. Osteoblasts, which differentiate from mesenchymal stem cells in the bone marrow and give rise to osteocytes, are responsible for bone formation $(6,7)$. By contrast, osteoclasts are derived from mononucleated hematopoietic progenitor cells and are specialized bone resorptive cells (8). Osteoblasts also secrete the receptor activator of nuclear factor- $\kappa \mathrm{B}$ ligand (RANKL), which is essential for osteoclast differentiation and regulates bone resorption in this manner $(9,10)$.

Bone marrow-derived mesenchymal stem cells (BMSCs) are important in maintaining the homeostasis of bone mass and are vital in the pathogenesis of osteoporosis $(11,12)$. The defective proliferation and osteogenic differentiation ability of BMSCs reduce bone mass in the process of osteoporosis. Several studies have shown that the osteogenic differentiation of BMSCs may be suppressed under stressful conditions, and finally lead to osteoporosis $(13,14)$. Osteoclast precursors differentiate into osteoclasts in the presence of monocyte colony stimulating factor and RANKL $(15,16)$. The formation and activity of osteoclasts are increased in osteoporosis (17). 
Shi et al reported that glucocorticoids exerted dose-dependent effects, which promote osteoclastogenesis in vivo and stimulate RANKL-induced osteoclast formation and function in vitro (18).

Ligusticum wallichii Franchat, also known as Chuanxiong, is considered one of the most widely used traditional Chinese medicines. Tetramethylpyrazine (TMP), the pharmacologically active component extracted from Chuanxiong, has been found to have anti-inflammatory, anticancer, anti-oxidative and anti-apoptotic effects in several types of cell (19-21). In our previous study, it was shown that TMP promoted the viability and inhibited the glucocorticoid-induced apoptosis of BMSCs (22). However, whether TMP can ameliorate the defective osteogenic differentiation of BMSCs in GIOP, and how TMP affects the formation and function of osteoclasts in GIOP remain to be elucidated.

In the present study, the protective effects of TMP on BMSC differentiation and osteoclast formation in GIOP were investigated in vivo and in vitro. The resulting data demonstrated that TMP promoted osteogenesis and inhibited osteoclastogenesis in GIOP rats, and indicated the inhibition of RANKL and IL-6 in BMSCs as a possible mechanism for the protective effects of TMP against glucocorticoid-induced osteoclastogenesis.

\section{Materials and methods}

Animals. A total of 20 4-month-old female Sprague-Dawley rats, weighing $217 \pm 15.5 \mathrm{~g}$, were obtained from the Experimental Animal Center at The Fourth Military Medical University (Xi'an, China), and were housed under specific pathogen-free conditions $\left(20^{\circ} \mathrm{C} ; 12\right.$-h light/dark cycles; $50-55 \%$ humidity $)$ with free access to food and water. The rats were administered intraperitoneally with either distilled water as the control group ( $\mathrm{n}=5$ ) or $2.5 \mathrm{mg} / \mathrm{kg}$ prednisolone (Sigma-Aldrich; Merck Millipore, Darmstadt, Germany) as the GIOP group ( $n=15)$ daily for 12 weeks. At 1 week following the first administration, the 15 rats in the GIOP group were randomly divided into three experimental groups, each containing five rats. The rats were injected intraperitoneally with either sesame oil (as a vehicle control), $5 \mathrm{mg} / \mathrm{kg}$ body weight of TMP or $20 \mathrm{mg} / \mathrm{kg}$ body weight of TMP (Sigma-Aldrich; Merck Millipore) daily for 12 weeks. The doses of TMP used were selected based on previous in vivo studies $(19,23)$. Subsequently, BMSCs were isolated from the rats, and the fourth lumbar vertebrae, distal femurs and blood samples were collected from the rats in the control and GIOP groups. No significant differences in total body weights were found among the groups prior to the rats being sacrificed. All experimental procedures involving animals were approved by the Ethics in Animal Research Committee of The Fourth Military Medical University (permission no. 20110405-5).

Micro-computed tomography (CT) analysis. The fourth lumbar vertebrae were scanned using an Explore Locus SP Pre-Clinical Specimen micro-CT (GE Healthcare Bio-Sciences, Pittsburgh, PA, USA) with $8 \mathrm{~mm}$ resolution, a $50 \mathrm{kV}$ tube voltage and a $0.1 \mathrm{~mA}$ tube current. Reconstruction and 3D quantitative analyses were performed using GEHC MicroView software, version 2.1 (GE Healthcare Bio-Sciences). Similar settings for scans and analyses were used for all samples. The trabecular bone region from the vertebral body was outlined for each micro-CT slice, which excluded the cranial and caudal endplate regions. The following 3D indices in the defined region of interest were analyzed: Bone mineral density (BMD), relative bone volume/total volume (BV/TV), trabecular number (Tb.N), trabecular thickness (Tb.Th), structure model index (SMI) and trabecular separation (Tb.Sp). The operator performing the scan analyses was blinded to the treatment procedures involving the specimens.

Histochemistry and immunohistochemistry. Following fixation for 2 days using $4 \%$ paraformaldehyde, the left femurs were transferred to $80 \%$ formic acid for decalcification for 14 days, embedded in paraffin and then cut into horizontal sections of 5- $\mu \mathrm{m}$ thickness. For tartrate-resistant acid phosphatase (TRAP) staining, the sections were reactivated in $0.2 \mathrm{M}$ Tris buffer and then stained using Acid Phosphatase Kit-387-A (Sigma-Aldrich; Merck Millipore) for $2 \mathrm{~h}$ at room temperature according to the manufacturer's protocol. For staining of osterix (OSX), the sections were incubated with anti-OSX primary antibody (ab22552, 1:100; Abcam, Cambridge, MA, USA) at $4^{\circ} \mathrm{C}$ overnight. All sections were observed and images were captured using a florescence microscope (Olympus BX-60; Olympus Corporation, Tokyo, Japan). For each sample, values represent five-stained sections of equivalent depth.

ELISA. The levels of bone degradation markers, serum C-telopeptide of type I collagen (CTX-1) and TRAP were measured in the blood samples of the rats using ELISA assay kits (Immunodiagnostic Systems, Ltd., Tyne \& Wear, UK) according to the manufacturer's protocol. BMSCs were seeded in 6-well plates at a density of $1 \times 10^{-6}$ cells/well. Following osteogenic induction with or without $10^{-6} \mathrm{M}$ dexamethasone (Dex; Sigma-Aldrich; Merck Millipore) at $37^{\circ} \mathrm{C}$ for 14 days, the BMSCs were incubated in serum-free medium with or without $\operatorname{TMP}(50,100$ or $200 \mu \mathrm{M})$ at $37^{\circ} \mathrm{C}$ for $48 \mathrm{~h}$. The expression levels of RANKL and IL-6 in the culture medium were measured using ELISA assay kits (R\&D Systems, Inc., Minneapolis, MN, USA) according to the manufacturer's protocol. Total protein concentrations were measured using a Bradford protein assay.

Osteogenic differentiation assay in BMSCs. The isolation and primary culture of BMSCs were performed as previously described (24), and the cells were characterized using MSC minimal criteria (25). Following osteogenic induction with $10^{-6} \mathrm{M}$ Dex for 14 days, the BMSCs were incubated with serum-free medium with or without TMP $(50,100$, or $200 \mu \mathrm{M}$ ) for $48 \mathrm{~h}$. Then the cells were stained using a BCIP/NBT Alkaline Phosphatase Color Development kit (Gibco; Thermo Fisher Scientific, Inc., Waltham, MA, USA). The activity of alkaline phosphatase (ALP) was detected as previously described (26) and calculated using absorbance measurements at $405 \mathrm{~nm}$. All sections were observed using an Olympus BX-60 microscope (Olympus Corporation). Following a 21-day period of osteogenic induction, Alizarin Red S staining was performed to detect calcium deposition, as previously described (27). The absorbance of the released Alizarin Red S was measured using a Thermo Labsystems Multiscan MK-3 enzyme-linked microplate reader (Thermo Fisher Scientific, Inc.) at a wavelength of $562 \mathrm{~nm}$. 
Reverse transcription-quantitative polymerase chain reaction (RT-qPCR) analysis. Total RNA was extracted from the cells using TRIzol reagent (Invitrogen; Thermo Fisher Scientific, Inc.). cDNA synthesis was performed using the Prime Script RT reagent kit (Takara Biotechnology Co., Ltd., Dalian, China). The RT-qPCR analysis was performed using a CFX96 (Bio-Rad Laboratories, Inc., Hercules, CA, USA) instrument. Individual reactions were conducted in 96-well optical reaction plates using SYBR Premix Ex Taq II (Tli RNaseH Plus) (Takara Biotechnology Co., Ltd.) as previously described (18). Amplifications were performed as follows: Initial denaturation at $95^{\circ} \mathrm{C}$ for $30 \mathrm{sec}$, followed by 40 cycles of denaturation at $95^{\circ} \mathrm{C}$ for $5 \mathrm{sec}$ and annealing at $58^{\circ} \mathrm{C}$ for $15 \mathrm{sec}$. The expression levels of target genes were normalized to the reference gene GAPDH. The $2^{-\Delta \Delta \mathrm{Cq}}$ method was applied to calculate the relative gene expression (28). The 5'-3' sequences of the forward and reverse primers were as follows: ALP, forward 5'-GTCCCACAAGAG CCCACAAT-3' and reverse 5'-CAACGGCAGAGCCAGGAA T-3'; collagen, type I, $\alpha 1$ (COL1A1), forward 5'-GACATGTTC AGCTTTGTGGACCTC-3' and reverse 5'-AGGGACCCTTAG GCCATTGTGTA; osteocalcin (OCN), 5'-CAGTAAGGTGGT GAATAGACTCCG-3' and reverse 5'-GGTGCCATAGAT GCGCTTG-3'; osterix (OSX), forward 5'-CACCCATTGCCA GTAATCTTCGT-3' and reverse 5'-GGACTGGAGCCATAG TGAGCTTCT-3'; TRAP, forward 5'-GCCTCTTGCGTCCTC TATGA-3' and reverse 5'-AGCACCATCCACGTATCCA-3'; nuclear factor of activated T-cells, cytoplasmic 1 (NFATC1), forward 5'-GCTCGCCTTTTCAACTTTCT-3' and reverse 5'-GCCTGGGACACACCTTTCTA-3; cathepsin K (CTSK), forward 5'-CGACTATCGAAAGAAAGGCTATG-3' and reverse 5'-AAAGCCCAACAGGAACCAC-3'; GAPDH, forward 5'-CCTGCACCACCAACTGCTTA-3' and reverse 5'-GGCCATCCACAGTCTTCTGAG-3'.

Detection of osteoclast formation in vitro. The hematopoietic mononucleated precursors of osteoclasts were isolated from the bone marrow and seeded in 6-well plates at a density of $1 \times 10^{-6}$ cells/well. Cells were cultured in the presence of $100 \mathrm{ng} / \mathrm{ml}$ RANKL and $10^{-6} \mathrm{M}$ Dex at $37^{\circ} \mathrm{C}$ for 7 days. Osteoclast formation was measured by quantifying the TRAP-positive stained cells. Briefly, the cells were fixed with $10 \%$ formalin for $10 \mathrm{~min}$ and ethanol/acetone (1:1) for $1 \mathrm{~min}$, and then stained using the Acid Phosphatase Kit-387-A (Sigma-Aldrich; Merck Millipore). The osteoclasts in each well were counted using a light microscope (Leica Microsystems GmbH, Wetzlar, Germany).

Statistical analysis. Statistical analyses were performed using SPSS software, version 15.0 (SPSS, Inc., Chicago, IL, USA). Quantitative data are presented as the mean \pm standard deviation and were compared using a one-way analysis of variance followed by a Bonferroni post-hoc test. $\mathrm{P}<0.05$ was considered to indicate a statistically significant difference.

\section{Results}

TMP improves osteoblast differentiation and osteoclast maturation in GIOP rats. To confirm the effects of TMP on bone mass and micro-architecture, a GIOP rat model was established. The fourth lumbar vertebrae were collected and scanned via micro-CT assessment. The analyses of the trabecular bone of the lumbar vertebrae indicated that excess glucocorticoids significantly reduced bone mass and deteriorated bone micro-architecture, as indicated by decreases in the BMD, BV/TV, Tb.N and Tb.Th, and increases in the SMI and Tb.Sp of the GIOP rats (Fig. 1A and B). Treatment of the GIOP rats with TMP partially ameliorated these bone parameters and improved the micro-architecture of the trabecular bone in the lumbar vertebrae. These results demonstrated that TMP protected the trabecular bone mass from excess exposure to glucocorticoids.

TMP promotes osteogenesis and inhibits osteoclastogenesis in GIOP rats in vivo. To investigate whether TMP affects osteogenesis and osteoclastogenesis in vivo, immunostaining and TRAP staining of distal femurs were performed. As shown in Fig. 2, glucocorticoids significantly reduced osteogenesis and enhanced osteoclastogenesis, compared with the control group. However, TMP treatment significantly increased the number of OSX-positive cells and decreased the number and spread of TRAP-positive cells (Fig. 2A-D). In addition, the activities of CTX-1 and TRAP were detected to measure the serum levels of osteoclastic markers. Compared with the control group, the activities of CTX-1 and TRAP were markedly elevated in the GIOP group. However, treatment with TMP significantly decreased the serum activities of CTX-1 and TRAP (Fig. 2E). These data suggested that treatment with TMP partially promoted osteogenesis and inhibited osteoclastogenesis in the GIOP rats.

Protection by TMP on osteogenic differentiation of BMSCs. To examine whether TMP promoted the osteogenic differentiation of BMSCs against excess glucocorticoids in vitro, the activity of ALP and calcium mineralization were examined, in addition to the mRNA expression levels of osteogenic genes, including ALP, COL1A1, OCN and OSX. A $10^{-6}$ MA concentration of Dex was used a high dose of glucocorticoids. As shown in Fig. 3A and B, $10^{-6} \mathrm{M}$ Dex significantly decreased the expression of ALP and the level of calcium mineralization, compared with the control group. However, the groups treated with TMP treatment exhibited significant increases in the activity of ALP and mineralization. In addition, TMP significantly elevated the expression levels of the osteogenic genes, compared with those in the Dex-only treated group (Fig. 4). Taken together, these data revealed that TMP improved the osteogenic differentiation of the BMSCs against excess glucocorticoids.

TMP inhibits osteoclastic differentiation in vitro. To examine whether TMP can protect osteoclast precursors against glucocorticoids in vitro, the present study examined osteoclast differentiation in the presence of excess glucocorticoids using TRAP staining. A previous study showed that high doses of Dex stimulated osteoclast formation in vitro (18), therefore, the osteoclasts in the present study were also exposed to $10^{-6} \mathrm{M}$ Dex. It was found that exposure to $10^{-6} \mathrm{M}$ Dex alone significantly increased the number of TRAP-positive cells (Fig. 5A and B). Treatment with different concentrations of TMP $(50,100$ or $200 \mu \mathrm{M})$ significantly reduced the numbers of TRAP-positive cells, compared with those in the group treated with Dex alone. The expression levels of several osteoclastogenesis-related genes were also investigated, including 
A

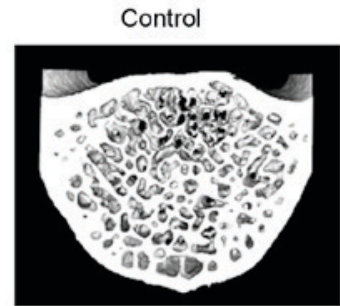

GIOP

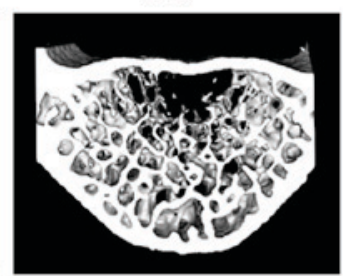

GIOP+TMP (5)

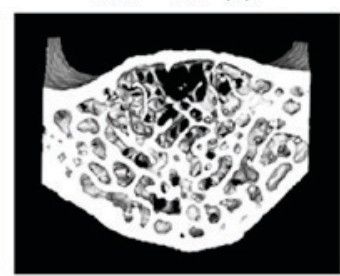

GIOP+TMP (20)

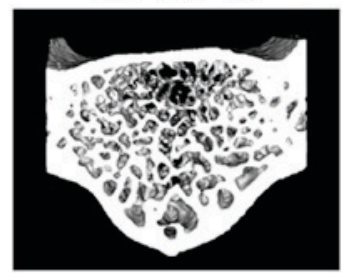

B
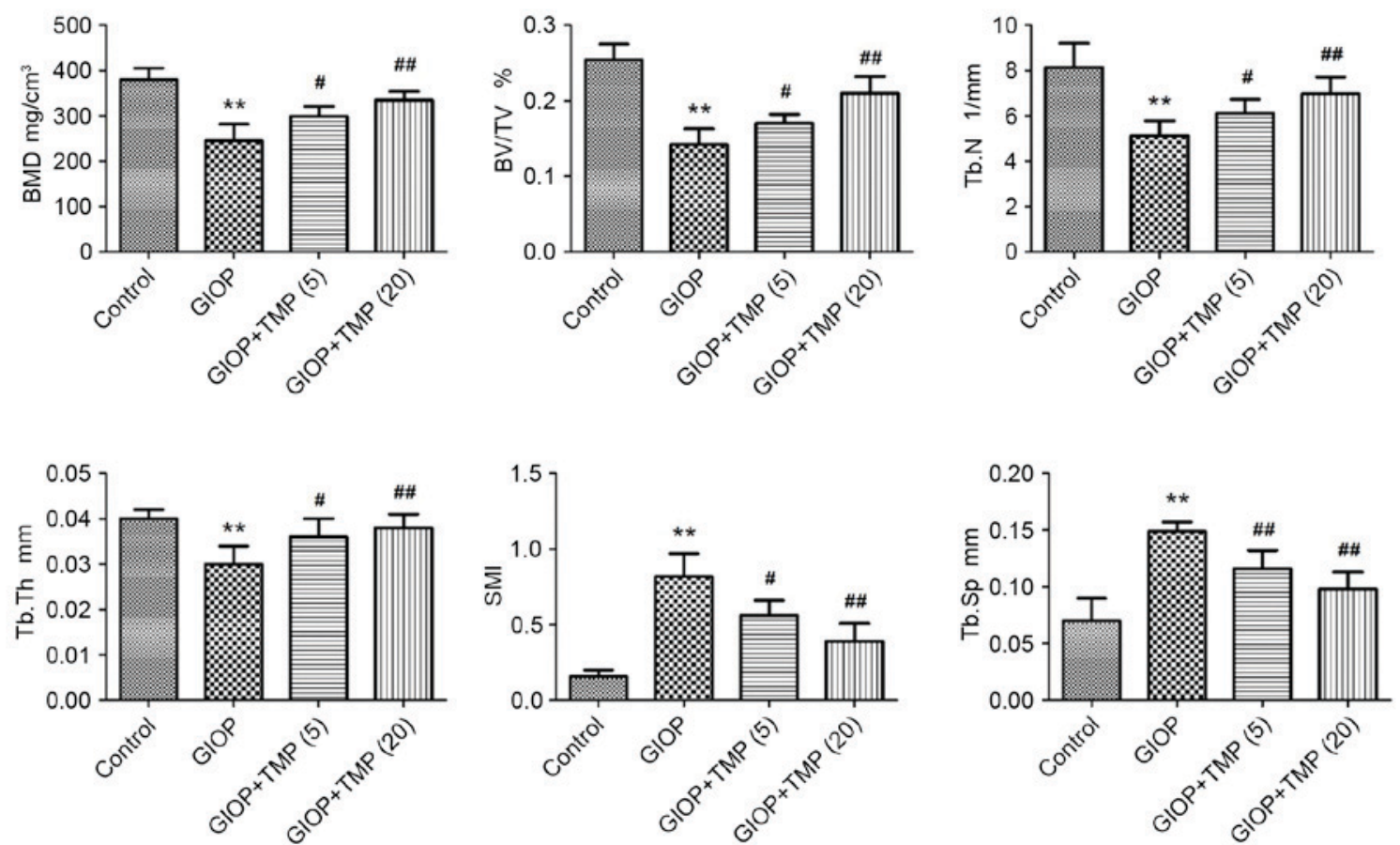

Figure 1. TMP improves trabecular bone mass in GIOP rats. (A) Micro-CT images within the fourth lumbar vertebrae region (magnification, $\mathrm{x} 40$ ). (B) Micro-CT analysis quantification within the fourth lumbar vertebrae region. The $3 \mathrm{D}$ indices in the defined region of interest were analyzed. " $\mathrm{P}<0.01$, vs. control group; ${ }^{\#} \mathrm{P}<0.05$ and ${ }^{\# \#} \mathrm{P}<0.01$, vs. GIOP group. (5) and (20) represent 5 and $20 \mathrm{mg} / \mathrm{kg}$ body weight of TMP, respectively. GIOP, glucocorticoid-induced osteoporosis; TMP, tetramethylpyrazine; BMD, bone mineral density; BV/TV, relative bone volume over the total volume; Tb.N, trabecular number; Tb.Th, trabecular thickness; SMI, structure model index; Tb.Sp, trabecular separation.

TRAP, NFATC1 and CTSK, using RT-qPCR analysis on day 3. Compared with the control group, all of the above genes were significantly upregulated in the group treated with Dex alone. However, treatment with all concentrations of TMP downregulated the expression of these genes, compared with those in the Dex-only treatment group (Fig. 5C). These data indicated that TMP acts as a potent inhibitor of osteoclastic differentiation under glucocorticoid exposure in vitro.

TMP inhibits the expression of RANKL and IL-6 in BMSCs. To investigate the possible reasons for the protective effects of TMP under Dex exposure, the present study measured the expression levels of RANKL and IL-6 in BMSCs using ELISA analysis. RANKL, which is originally formed by osteoblasts, binds to RANK on osteoclasts and results in osteoclast activation. IL-6 also acts as an activator of osteoclast formation. The expression levels of these two cytokines were significantly elevated by Dex treatment. However, when different concentrations of TMP were administered to the BMSCs, the levels of RANKL and IL-6 were significantly downregulated (Fig. 6). These results suggested that the inhibition of RANKL and
IL-6 in BMSCs is a possible mechanism for the protective effects of TMP against Dex-induced osteoclastogenesis.

\section{Discussion}

The survival and function of BMSCs are essential for the maintenance of bone tissue homeostasis, as are osteoclasts. In our previous study, it was found that the viability of BMSCs derived from GIOP rats was decreased, and that TMP protected the BMSCs by inhibiting apoptosis in the GIOP state $(22,29)$. The present study further investigated the effects of TMP on the osteogenic function of BMSCs and osteoclast formation to provide evidence supporting the potential application of TMP in the prevention and treatment of GIOP.

Long-term glucocorticoid therapy results primarily in trabecular bone loss in patients with GIOP. As GIOP rats share similar features with patients with GIOP, the micro-architecture of the fourth lumbar vertebrae in GIOP rats adequately reflects the change of bone mass in GIOP. Previous static bone histomorphometric analysis has shown that trabecular bone mass is markedly decreased in GIOP rats (30), which 
$A$

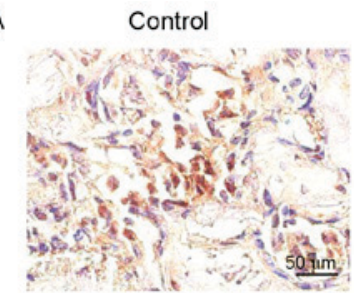

B

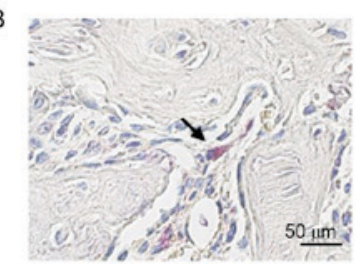

C

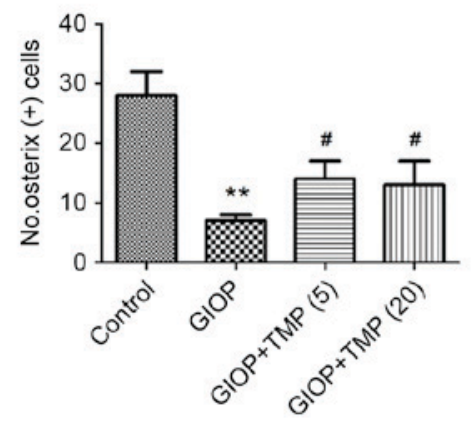

GIOP
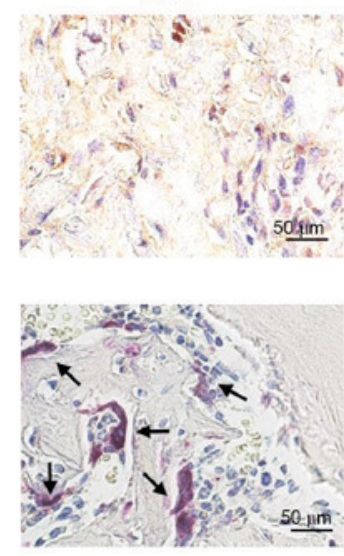

D
GIOP+TMP (5)
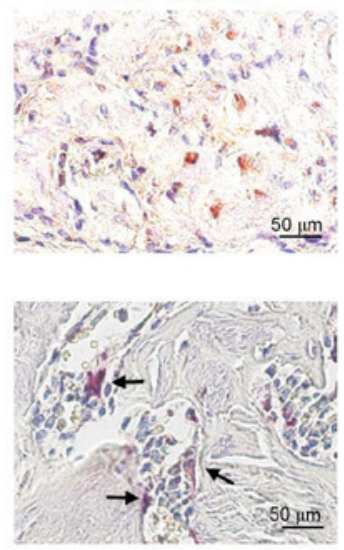

GIOP+TMP (20)
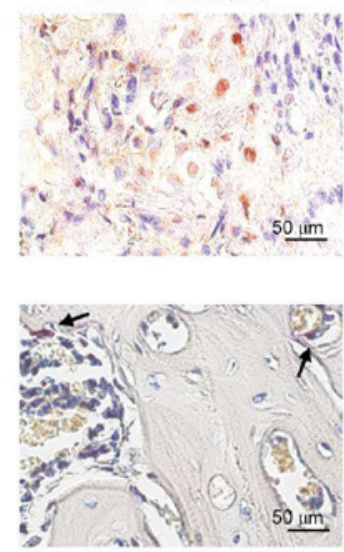
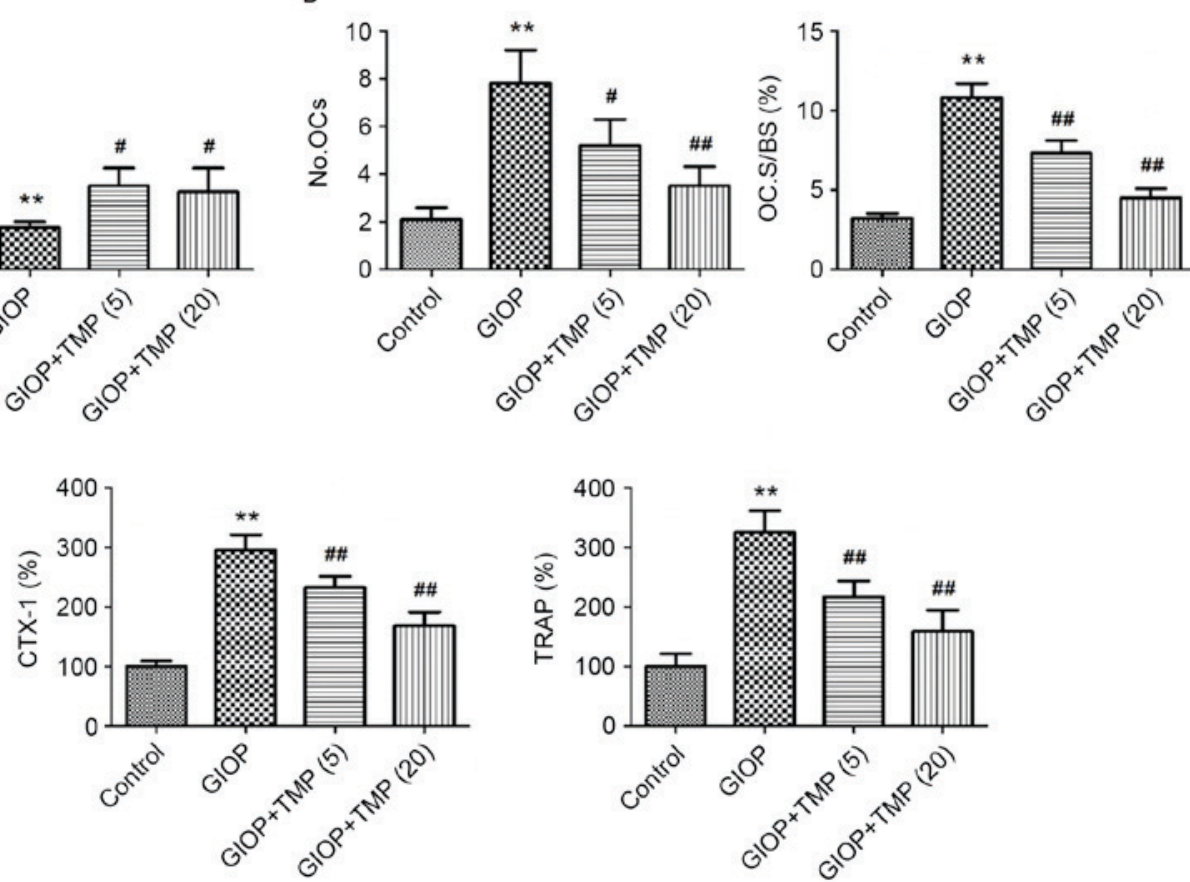

Figure 2. TMP promotes osteogenesis and inhibits osteoclastogenesis in vivo. (A) Representative staining (brown) of osterix. (B) TRAP staining of distal femurs. Arrows indicate areas of TRAP-positive multinuclear cells. (C) Quantification of osterix-positive cells. (D) TRAP-positive multinuclear ( $\geq 3$ ) cells of each group were counted. (E) Serum levels of CTX-1 and TRAP in rats. The control value of CTX-1 was $25.71 \pm 2.37 \mathrm{ng} / \mathrm{ml}$ and the control value of TRAP was $19.36 \pm 2.55 \mathrm{U} / \mathrm{ml} .{ }^{* *} \mathrm{P}<0.01$, vs. control group; ${ }^{\#} \mathrm{P}<0.05$ and ${ }^{\# \#} \mathrm{P}<0.01$, vs. GIOP group (n=5). (5) and (20) represent 5 and $20 \mathrm{mg} / \mathrm{kg}$ body weight of TMP, respectively. GIOP, glucocorticoid-induced osteoporosis; TMP, tetramethylpyrazine; OC, osteoclasts; OC.S/BS, osteoclast surface/bone surface; TRAP, tartrate-resistant acid phosphatase.

is consistent with the data obtained in the present study. It has also been reported that TMP therapy exerts positive effects in a rat model of Parkinson's disease and spinal cord injury $(23,31)$. In the present study, it was confirmed that TMP treatment ameliorated the decreased trabecular bone mass of the lumbar vertebrae in the GIOP rats, and it may be a suitable candidate for the prevention and treatment of GIOP. Of note, it was found that treatment with TMP significantly increased the number of OSX-positive cells and decreased the number of TRAP-positive cells in vivo, which suggested that TMP affected osteogenesis and osteoclastogenesis in the GIOP rats.

Although Dex is commonly used in the cell culture medium to differentiate BMSCs into different types of mature cells, the effects of different concentrations of Dex on BMSCs have been reported to vary. Gao et al and others reported that
$10^{-6} \mathrm{M}$ Dex had negative effects on BMSCs obtained from mice or rats $(29,32)$. In the present study, $10^{-6} \mathrm{M}$ Dex was also used to generate an in vitro model of excess glucocorticoid exposure stress. The resulting data demonstrated that $10^{-6} \mathrm{M}$ Dex caused a decline in cellular ALP activity, calcium mineralization and the expression of osteogenic genes, which was consistent with the in vivo results showing decreased osteogenesis in the GIOP rats. However, TMP partially reversed the inhibitory effect of Dex on BMSC osteogenic differentiation. These results suggested that TMP not only improved the viability of BMSCs, but also ameliorated the defective osteogenic differentiation of BMSCs, contributing to the promotion of osteogenesis in GIOP.

The effects of osteoclasts in GIOP, and the effects of glucocorticoids on osteoclast formation and function remain 
A
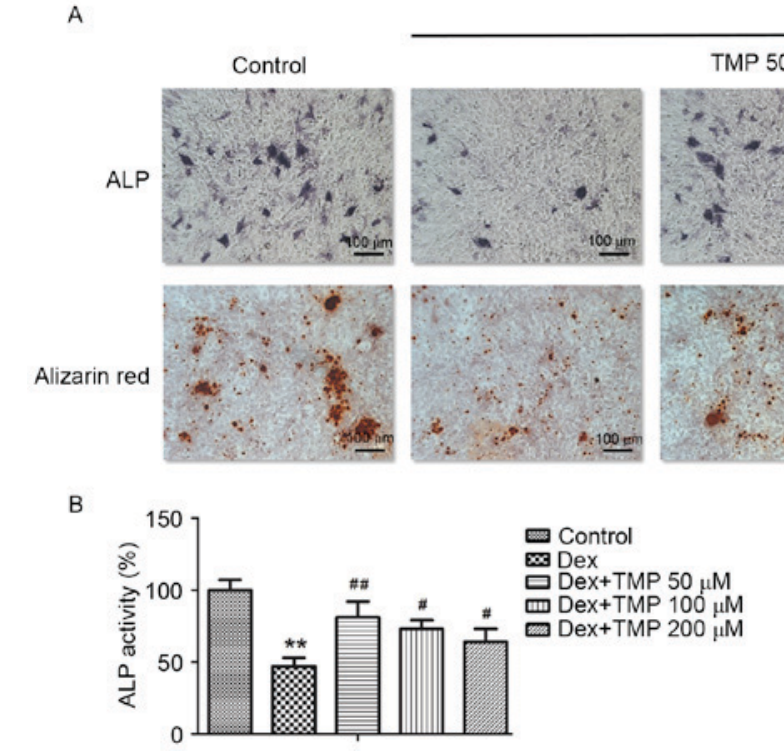

TMP $50 \mu \mathrm{M}$

$\operatorname{Dex} 10^{-6} \mathrm{M}$

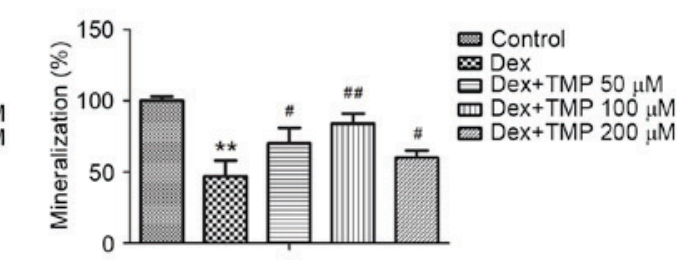

Figure 3. TMP promotes osteogenic differentiation of BMSCs in the presence of excess glucocorticoids in vitro. (A) Images of ALP staining and Alizarin Red S staining. (B) Activity of ALP and mineralization of BMSCs were calculated. The control value for the activity of ALP in BMSCs was $0.49 \pm 0.06 \mathrm{U} / \mathrm{mg}$ protein ${ }^{* *} \mathrm{P}<0.01$ vs. the control group; ${ }^{\#} \mathrm{P}<0.05$ and ${ }^{\# \#} \mathrm{P}<0.01$ vs. the Dex group $(\mathrm{n}=5)$. ALP, alkaline phosphatase; Dex, dexamethasone; TMP, tetramethylpyrazine.
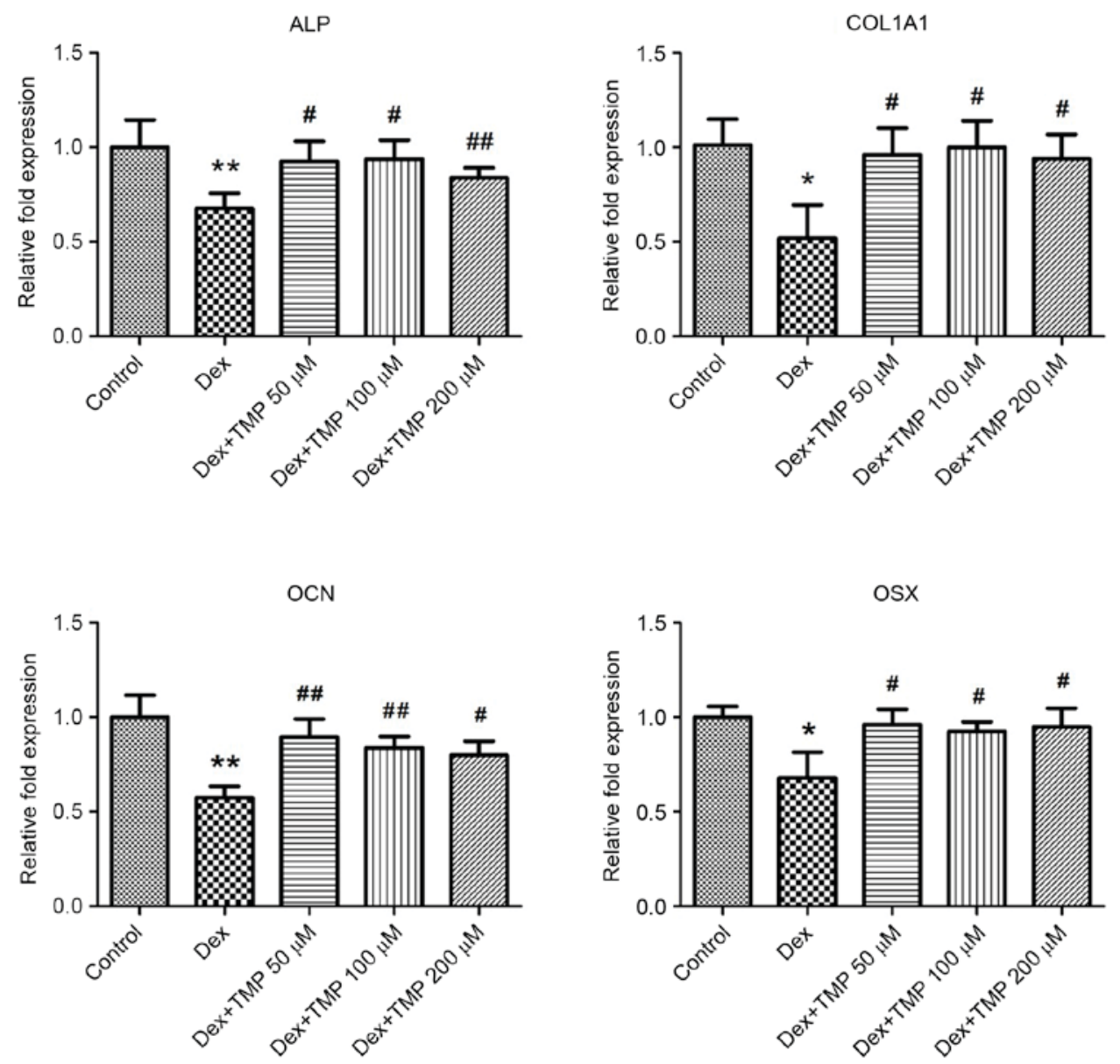

Figure 4. TMP elevates the mRNA expression levels of osteogenic genes. Expression of GAPDH served as a control. " $\mathrm{P}<0.05$ and ${ }^{* * *} \mathrm{P}<0.01$, vs. control group; ${ }^{\#} \mathrm{P}<0.05$ and ${ }^{\# \#} \mathrm{P}<0.01$, vs. Dex group $(\mathrm{n}=5)$. ALP, alkaline phosphatase; COL1A1, collagen, type I, $\alpha 1$; OCN, osteocalcin; OSX, osterix; Dex, dexamethasone; TMP, tetramethylpyrazine.

to be fully elucidated. Kim et al documented that glucocorticoids inhibited the proliferation of osteoclast precursors in vitro (33). Shi et al found that high doses of glucocorticoids promoted osteoclastogenesis, whereas low doses had no such 
A

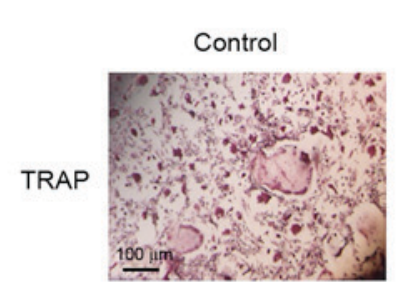

B

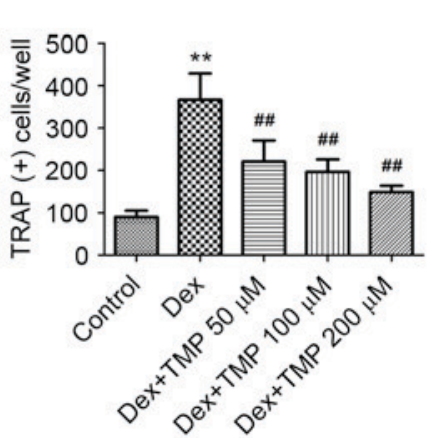

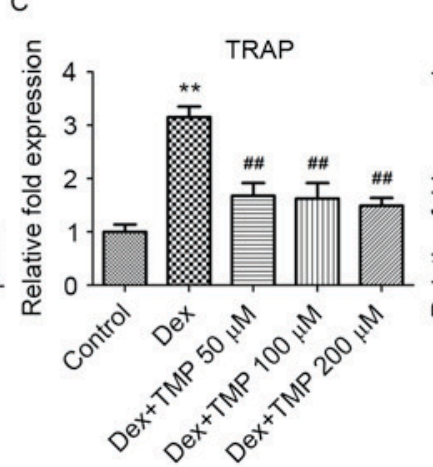
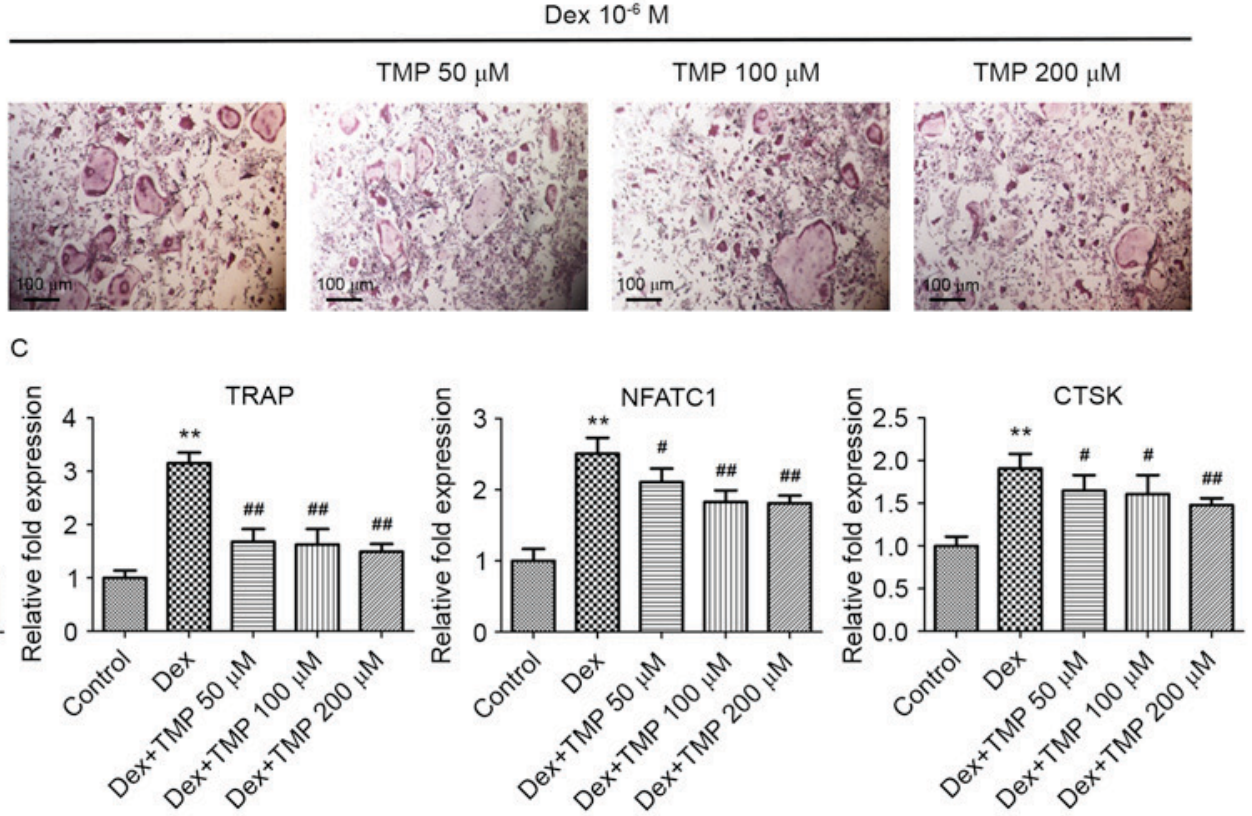

Figure 5. TMP inhibits osteoclastic differentiation under Dex exposure in vitro. (A) TRAP staining of osteoclasts treated with different concentrations of TMP in the presence of $10^{-6} \mathrm{M}$ Dex. (B) Number of TRAP-positive multinuclear ( $\geq 3$ ) cells. (C) mRNA expression levels of osteoclastogenesis-related genes. Expression of GAPDH served as control. ${ }^{* *} \mathrm{P}<0.01$ vs. control group; ${ }^{\#} \mathrm{P}<0.05$ and ${ }^{\# \#} \mathrm{P}<0.01$, vs. Dex group ( $\mathrm{n}=5$ ). TRAP, tartrate resistant acid phosphatase; Dex, dexamethasone; TMP, tetramethylpyrazine; NFATC1, nuclear factor of activated T-cells, cytoplasmic 1; CTSK, cathepsin K.
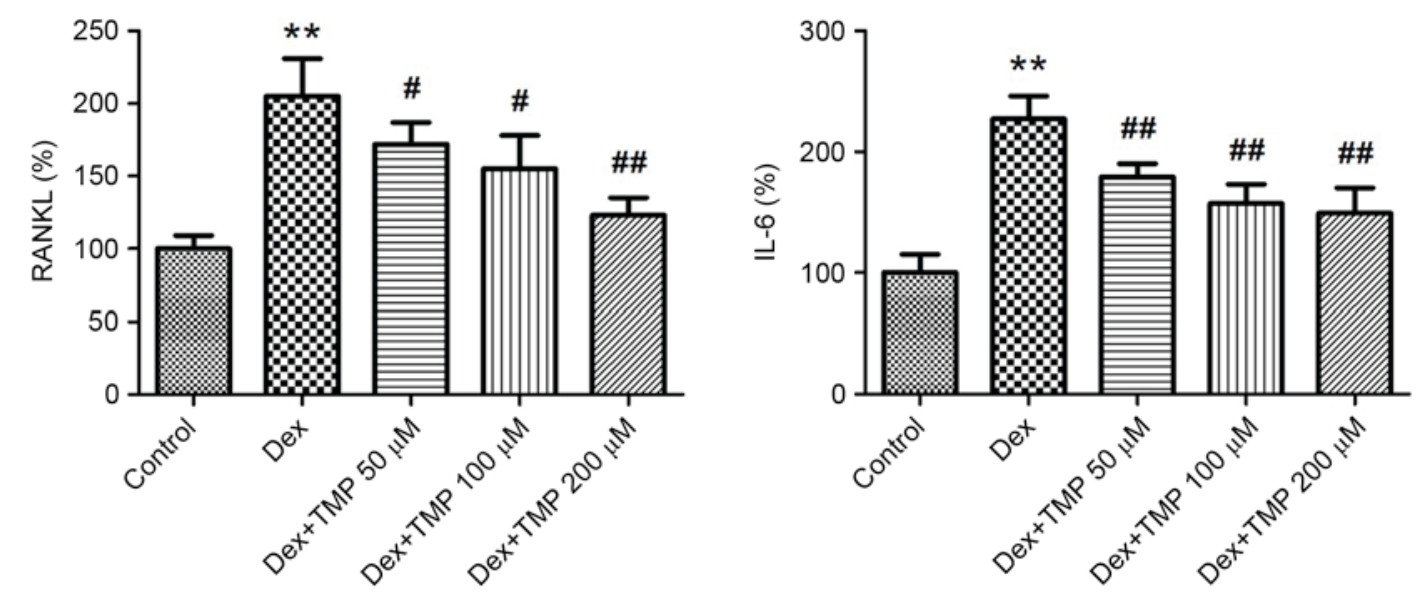

Figure 6. TMP inhibits expression levels of RANKL and IL-6 in BMSCs in the presence of Dex. Expression levels of RANKL and IL-6 in BMSCs are shown. The control value of RANKL was $3.25 \pm 0.27 \mathrm{ng} / \mathrm{mg}$ and the control of IL-6 was $1.83 \pm 0.19 \mathrm{ng} / \mathrm{mg}$. ${ }^{* *} \mathrm{P}<0.01$, vs. control group; ${ }^{\#} \mathrm{P}<0.05 \mathrm{and}{ }^{\# \#} \mathrm{P}<0.01$, vs. Dex group $(n=5)$. RANKL, receptor activator for nuclear factor- $\kappa$ B ligand; IL-6, interleukin-6; Dex, dexamethasone; TMP, tetramethylpyrazine.

effects (18). The data obtained in the present study showed that $10^{-6} \mathrm{M}$ Dex markedly promoted osteoclast formation in vitro, and TMP decreased osteoclast numbers and the expression of osteoclast-specific genes under Dex exposure. The inhibition of osteoclastogenesis in the presence of glucocorticoids may account for the anti-osteoporotic effects of TMP.

The RANKL secreted by osteoblasts, which belongs to the tumor necrosis factor superfamily, regulates osteoclast differentiation and leads to bone resorptive activities. Osteoblasts also secrete IL-6, which affects the expression of RANKL, enhances osteoclastogenesis and is implicated in the pathogenesis of osteoporosis (34). It has been reported that the enhancement of osteoclastogenesis in GIOP may be attributed to the upregulated expression of RANKL caused by glucocorticoids (35). The findings of the present study are consistent with this, which showed that TMP inhibited the generation of RANKL and IL-6 in BMSCs exposed to glucocorticoids following osteogenic induction. This data suggested that TMP may indirectly affect osteoclasts by decreasing the expression of RANKL and IL- 6 in BMSCs, and partly explains why TMP reduces osteoclastogenesis in GIOP. There are additional mechanisms accounting for the effects of TMP on BMSCs and osteoclasts. Several studies have reported that TMP decreases the production of reactive oxygen species, and protects cells against toxicity and oxidative stress $(20,23)$. In addition to regulating RANKL and IL-6, the anti-oxidative property of TMP may be involved in its anti-GIOP effects, of which further investigation is required. 
In conclusion, the present study investigated whether TMP had an effect on the osteogenic differentiation of BMSCs and formation of osteoclasts following exposure to excess glucocorticoids in vivo and in vitro. The results showed that TMP downregulated RANKL and IL-6, promoted osteogenesis and inhibited osteoclastogenesis to ameliorate the change in bone mass in the GIOP state. These results suggested that TMP may be a promising drug for the prevention and treatment of GIOP.

\section{Acknowledgements}

This study was supported by the National Natural Science Foundation of China (grant nos. 81572192 and 81472043) and the Program for Changjiang Scholars and Innovative Research Team in University (grant no. IRT13051).

\section{References}

1. Hofbauer LC, Hamann C and Ebeling PR: Approach to the patient with secondary osteoporosis. Eur J Endocrinol 162: 1009-1020, 2010.

2. Rizzoli R and Biver E: Glucocorticoid-induced osteoporosis: Who to treat with what agent? Nat Rev Rheumatol 11: 98-109, 2015 .

3. Canalis E, Mazziotti G, Giustina A and Bilezikian JP: Glucocorticoid-induced osteoporosis: Pathophysiology and therapy. Osteoporos Int 18: 1319-1328, 2007.

4. O'Brien CA, Jia D, Plotkin LI, Bellido T, Powers CC, Stewart SA, Manolagas SC and Weinstein RS: Glucocorticoids act directly on osteoblasts and osteocytes to induce their apoptosis and reduce bone formation and strength. Endocrinology 145: 1835-1841, 2004.

5. Jia D, O'Brien CA, Stewart SA, Manolagas SC and Weinstein RS: Glucocorticoids act directly on osteoclasts to increase their life span and reduce bone density. Endocrinology 147: 5592-5599, 2006.

6. Harada S and Rodan GA: Control of osteoblast function and regulation of bone mass. Nature 423: 349-355, 2003.

7. Karsenty G and Wagner EF: Reaching a genetic and molecular understanding of skeletal development. Dev Cell 2: 389-406, 2002.

8. Ash P, Loutit JF and Townsend KM: Osteoclasts derived from haematopoietic stem cells. Nature 283: 669-670, 1980.

9. Lacey DL, Timms E, Tan HL, Kelley MJ, Dunstan CR, Burgess T, Elliott R, Colombero A, Elliott G, Scully S, et al: Osteoprotegerin ligand is a cytokine that regulates osteoclast differentiation and activation. Cell 93: 165-176, 1998.

10. Lagasse E and Weissman IL: Enforced expression of Bcl-2 in monocytes rescues macrophages and partially reverses osteopetrosis in op/op mice. Cell 89: 1021-1031, 1997.

11. Bonyadi M, Waldman SD, Liu D, Aubin JE, Grynpas MD and Stanford WL: Mesenchymal progenitor self-renewal deficiency leads to age-dependent osteoporosis in Sca-1/Ly-6A null mice. Proc Natl Acad Sci USA 100: 5840-5845, 2003.

12. Miura M, Chen XD, Allen MR, Bi Y, Gronthos S, Seo BM, Lakhani S, Flavell RA, Feng XH, Robey PG, et al: A crucial role of caspase-3 in osteogenic differentiation of bone marrow stromal stem cells. J Clin Invest 114: 1704-1713, 2004.

13. Chen TL: Inhibition of growth and differentiation of osteoprogenitors in mouse bone marrow stromal cell cultures by increased donor age and glucocorticoid treatment. Bone 35: 83-95, 2004.

14. Huang Q, Shi J, Gao B, Zhang HY, Fan J, Li XJ, Fan JZ, Han YH, Zhang JK, Yang L, et al: Gastrodin: An ancient Chinese herbal medicine as a source for anti-osteoporosis agents via reducing reactive oxygen species. Bone 73: 132-144, 2015.

15. Boyce BF: Advances in osteoclast biology reveal potential new drug targets and new roles for osteoclasts. J Bone Miner Res 28: 711-722, 2013.

16. Wang K, Niu J, Kim H and Kolattukudy PE: Osteoclast precursor differentiation by MCPIP via oxidative stress, endoplasmic reticulum stress, and autophagy. J Mol Cell Biol 3: 360-368, 2011.
17. Xiu Y, Xu H, Zhao C, Li J, Morita Y, Yao Z, Xing L and Boyce BF: Chloroquine reduces osteoclastogenesis in murine osteoporosis by preventing TRAF3 degradation. J Clin Invest 124: 297-310, 2014.

18. Shi J, Wang L, Zhang H, Jie Q, Li X, Shi Q, Huang Q, Gao B, Han Y, Guo K, et al: Glucocorticoids: Dose-related effects on osteoclast formation and function via reactive oxygen species and autophagy. Bone 79: 222-232, 2015.

19. Chen L, Wei X, Hou Y, Liu X, Li S, Sun B, Liu X and Liu H: Tetramethylpyrazine analogue CXC195 protects against cerebral ischemia/reperfusion-induced apoptosis through PI3K/Akt/GSK3 $\beta$ pathway in rats. Neurochem Int 66: 27-32, 2014.

20. Gong X, Ivanov VN, Davidson MM and Hei TK: Tetramethylpyrazine (TMP) protects against sodium arsenite-induced nephrotoxicity by suppressing ROS production, mitochondrial dysfunction, pro-inflammatory signaling pathways and programed cell death. Arch Toxicol 89: 1057-1070, 2015.

21. Yu N, Zhang Z, Chen P, Zhong Y, Cai X, Hu H, Yang Y, Zhang J, $\mathrm{Li} \mathrm{K}, \mathrm{Ge} \mathrm{J}$, et al: Tetramethylpyrazine (TMP), an active ingredient of Chinese herb medicine Chuanxiong, attenuates the degeneration of trabecular meshwork through SDF-1/CXCR4 axis. PLoS One 10: e0133055, 2015.

22. Wang L, Zhang HY, Gao B, Shi J, Huang Q, Han YH, Hu YQ, Lu WG, Zhao ZJ, Liu BH, et al: Tetramethylpyrazine protects against glucocorticoid-induced apoptosis by promoting autophagy in mesenchymal stem cells and improves bone mass in glucocorticoid-induced osteoporosis rats. Stem Cells Dev 26: 419-430, 2017.

23. Lu C, Zhang J, Shi X, Miao S, Bi L, Zhang S, Yang Q, Zhou X, Zhang M, Xie Y, et al: Neuroprotective effects of tetramethylpyrazine against dopaminergic neuron injury in a rat model of Parkinson's disease induced by MPTP. Int J Biol Sci 10: 350-357, 2014.

24. Bouffi C, Bony C, Courties G, Jorgensen C and Noël D: IL-6-dependent PGE2 secretion by mesenchymal stem cells inhibits local inflammation in experimental arthritis. PLoS One 5: e14247, 2010.

25. Dominici M, Le Blanc K, Mueller I, Slaper-Cortenbach I, Marini F, Krause D, Deans R, Keating A, Prockop Dj and Horwitz E: Minimal criteria for defining multipotent mesenchymal stromal cells. The International society for cellular therapy position statement. Cytotherapy 8: 315-317, 2006.

26. Wang X, Harimoto K, Liu J, Guo J, Hinshaw S, Chang Z and Wang Z: Spata4 promotes osteoblast differentiation through Erk-activated Runx2 pathway. J Bone Miner Res 26: 1964-1973, 2011.

27. Chen JJ, Zhang NF, Mao GX, He XB, Zhan YC, Deng HB, Song DQ, Li DD, Li ZR, Si SY, et al: Salidroside stimulates osteoblast differentiation through BMP signaling pathway. Food Chem Toxicol 62: 499-505, 2013.

28. Livak KJ and Schmittgen TD: Analysis of relative gene expression data using real-time quantitative PCR and the 2(-Delta Delta C(T)) method. Methods 25: 402-408, 2001.

29. Wang L, Fan J, Lin YS, Guo YS, Gao B, Shi QY, Wei BY, Chen L, Yang L, Liu J and Luo ZJ: Glucocorticoids induce autophagy in rat bone marrow mesenchymal stem cells. Mol Med Rep 11: 2711-2716, 2015.

30. Cui L, Li T, Liu Y, Zhou L, Li P, Xu B, Huang L, Chen Y, Liu Y, Tian X, et al: Salvianolic acid B prevents bone loss in prednisone-treated rats through stimulation of osteogenesis and bone marrow angiogenesis. PLoS One 7: e34647, 2012.

31. Hu J, Cao Y, Wu T, Li D and Lu H: Micro-CT as a tool to investigate the efficacy of tetramethylpyrazine in a rat spinal cord injury model. Spine (Phila Pa 1976) 41: 1272-1278, 2016.

32. Gao B, Huang Q, Jie Q, Zhang HY, Wang L, Guo YS, Sun Z, Wei BY, Han YH, Liu J, et al: Ginsenoside-Rb2 inhibits dexamethasone-induced apoptosis through promotion of GPR120 induction in bone marrow-derived mesenchymal stem cells. Stem Cells Dev 24: 781-790, 2015.

33. Kim HJ, Zhao H, Kitaura H, Bhattacharyya S, Brewer JA, Muglia LJ, Ross FP and Teitelbaum SL: Glucocorticoids suppress bone formation via the osteoclast. J Clin Invest 116: 2152-2160, 2006.

34. Papanicolaou DA and Vgontzas AN: Interleukin-6: The endocrine cytokine. J Clin Endocrinol Metab 85: 1331-1333, 2000.

35. Tanaka Y: Glucocorticoid and bone metabolism and disease. Clin Calcium 23: 229-235, 2013 (In Japanese). 